\title{
ON THE CLASSIFICATION OF REAL MONO-GERMS OF CORANK ONE AND CODIMENSION ONE
}

\author{
KEVIN HOUSTON \\ School of Mathematics, University of Leeds \\ Leeds, LS2 9JT, U.K. \\ e-mail: k.houston@leeds.ac.uk
}

\begin{abstract}
Corank one mono-germs $f:\left(\mathbb{R}^{n}, 0\right) \rightarrow\left(\mathbb{R}^{p}, 0\right), n<p$, of $\mathcal{A}_{e}$-codimension one are classified by giving an explicit normal form.

1. Introduction. Classification of singularities up to $\mathcal{A}$-equivalence - up to local diffeomorphism in the source and target - is a primary goal of Singularity Theory. Recent work has been done in the classification of $\mathcal{A}_{e}$-codimension 1 singularities in [1], [2] and [4]. In [5] $\mathcal{A}_{e}$-codimension 1 corank 1 multi-germs $f:\left(\mathbb{C}^{n}, S\right) \rightarrow\left(\mathbb{C}^{p}, 0\right)$ with $n<p$ and $S$ a finite set have been classified. Explicit normal forms for the mono-germs are given and constructions involving three operations on these mono-germs are used to produce the multi-germs. In this paper we shall give an explicit description for the real versions of the mono-germs.
\end{abstract}

In contrast to the complex case, real $\mathcal{K}$-equivalent corank $1 \mathcal{A}_{e^{-c o d i m e n s i o n ~}} 1$ mapgerms are not $\mathcal{A}$-equivalent. For example, consider $(x, y) \mapsto\left(x, y^{2}, y^{3} \pm x^{2} y\right)$, both germs have $\mathcal{A}_{e}$-codimension 1 , are $\mathcal{K}$-equivalent but not $\mathcal{A}$-equivalent, see $[9]$. Yet, over the complex numbers the two are equivalent.

The key comes from the observation that the inequivalent maps arise from the augmentation of the map $y \mapsto\left(y^{2}, y^{3}\right)$ unfolded by $\left(\lambda, y^{2}, y^{3}+\lambda y\right)$ or $\left(\lambda, y^{2}, y^{3}-\lambda y\right)$. Note that any $\mathcal{A}_{e^{-c o d i m e n s i o n}} 1$ map $\mathcal{K}$-equivalent to $y \mapsto\left(y^{2}, y^{3}\right)$ is $\mathcal{A}$-equivalent to it. A corollary of the classification given here is that if $f$ is not an augmentation then any corank 1 $\mathcal{A}_{e}$-codimension 1 map $\mathcal{K}$-equivalent to it is also $\mathcal{A}$-equivalent. It would be interesting to know how general a phenomenon this is for primitive mono-germs.

In [1] Cooper shows how to classify real $\mathcal{A}_{e}$-codimension 1 corank 1 mono-germs by explicit coordinate changes in the source and target of the map in the case of $p=n+1$. In a similar way one could classify corank 1 real mono-germs for $n<p$. Instead, in Section 3 we present a significantly simpler method of classifying mono-germs by using left and right

2000 Mathematics Subject Classification: 58K40.

The paper is in final form and no version of it will be published elsewhere. 
diffeomorphisms in source and target and utilising information on the defining equations of the multiple point spaces of the complexified map.

As expected the case of real multi-germs is more complicated than the complex case. This is because one of the processes used to create $\mathcal{A}_{e}$-codimension 1 multi-germs from unfolding other codimension 1 germs, the process of binary concatentation (see [2] or [5]), depends on the unfoldings used. Nonetheless, it seems likely that real multi-germs have a form similar to that in the complex case as described in Theorem 6.4 of [5].

2. Augmentation. First we give some notation used throughout the paper. The field $\mathbb{K}$ will denote either the complex or real numbers, $\mathbb{C}$ and $\mathbb{R}$ respectively. Map-germs will be smooth over the reals and analytic when over the complexes. The multiplicity of a map will be denoted mult $(f)$. The $\mathcal{A}_{e}$-codimension of a map-germ $f$ will be denoted $\operatorname{cod}(f)$. If two germs $f$ and $g$ are $\mathcal{A}$-equivalent then we use the notation $f \sim_{\mathcal{A}} g$. The set $S$ will be a finite set of points and usually they will be the origins of a collection of copies of $\mathbb{K}^{n}$ for some $n$. In this section $n$ and $p$ will be quite general, only later will we assume $n<p$. Other notation will be the standard used in Singularity Theory, see [10].

First we define the positive and negative augmentation of a map-germ.

Definition 2.1. Let $f:\left(\mathbb{K}^{n}, 0\right) \rightarrow\left(\mathbb{K}^{p}, 0\right)$ be a map with a 1 -parameter stable unfolding $F:\left(\mathbb{K}^{n} \times \mathbb{K}, 0\right) \rightarrow\left(\mathbb{K}^{p} \times \mathbb{K}, 0\right)$, where $F(x, \lambda)=\left(f_{\lambda}(x), \lambda\right)$. Then the positive augmentation of $f$ by $F$ is the map $A_{F}^{+}(f)$ given by $(x, \lambda) \mapsto\left(f_{\lambda^{2}}(x), \lambda\right)$. The negative augmentation of $f$ by $F$ is the map $A_{F}^{-}(f)$ given by $(x, \lambda) \mapsto\left(f_{-\lambda^{2}}(x), \lambda\right)$.

The image of the example of the standard cusp $x \mapsto\left(x^{2}, x^{3}\right)$ unfolded by $\left(x^{2}, x^{3}-\lambda x, \lambda\right)$ and augmented to $\left(x^{2}, x^{3}-\lambda^{2} x, \lambda\right)$ is shown in Figure 1.
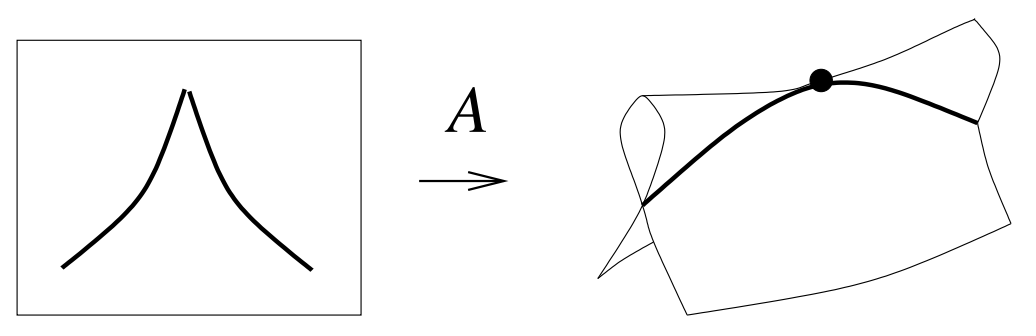

Figure 1. Augmentation of a cusp

TheOrem 2.2 (See $[2])$. Suppose $f:\left(\mathbb{K}^{n}, 0\right) \rightarrow\left(\mathbb{K}^{p}, 0\right)$ is a finitely determined mapgerm with a 1-parameter stable unfolding. Then $\operatorname{cod}\left(A_{F}^{ \pm}(f)\right)=\operatorname{cod}(f)$.

Thus we can produce new codimension 1 maps from old ones. If $f$ is not $\mathcal{A}$-equivalent to the augmentation of another germ then $f$ is called primitive.

Via the following lemma we are in a position to reduce $\mathcal{A}$-equivalence problems to $\mathcal{K}$-equivalence ones.

Lemma 2.3. Suppose $f_{t}:\left(\mathbb{K}^{n}, 0\right) \rightarrow\left(\mathbb{K}^{p}, 0\right), t \in[0,1]$, is a smooth family of primitive $\mathcal{A}_{e}$-codimension 1 multi-germs. If the $f_{t}$ are $\mathcal{K}$-equivalent for all $t$, then they are $\mathcal{A}$-equivalent for all t. 
Proof. We use Mather's Lemma ([8], Lemma 3.1). Condition (b) of his lemma is satisfied since every member of the family has $\mathcal{A}_{e}$-codimension 1 and so $\mathcal{A}$-codimension is constant also (see [10], Proposition 4.5.2).

Since $f_{t}$ is primitive for all $t$ the $\mathcal{A}$-orbit of $f_{t}$ is open in its $\mathcal{K}$-orbit, as can be seen from the proof of Lemma 3.6 of [5]. As the family members are all $\mathcal{K}$-equivalent this implies Mather's condition (a). Hence the family is contained in a single $\mathcal{A}$-orbit.

Suppose that $F$ and $\widetilde{F}$ are two 1-parameter stable unfoldings of the map $f:\left(\mathbb{K}^{n}, 0\right) \rightarrow$ $\left(\mathbb{K}^{p}, 0\right)$. Then $F(x, \lambda)$ is $\mathcal{A}$-equivalent to $\widetilde{F}(x, \phi(\lambda))$ for some smooth $\phi:(\mathbb{K}, 0) \rightarrow(\mathbb{K}, 0)$.

Theorem 2.4. Suppose $\mathbb{K}=\mathbb{R}$. Then $A_{\widetilde{F}}^{+}(f) \sim_{\mathcal{A}} A_{F}^{\operatorname{sign}(d \phi(0))}(f)$.

Proof. The proof is the same as Proposition 2.1 in [2] except their $\alpha\left(t^{2}\right)= \pm \beta(t)^{2}$ as we are working over the reals.

In the case of $\mathbb{K}=\mathbb{C}$ it has already been shown that an augmentation is independent up to $\mathcal{A}$-equivalence of the unfolding used.

Corollary 2.5. Suppose $f \sim_{\mathcal{A}} f^{\prime}$. Then $f$ and $f^{\prime}$ can be induced from the same stable unfolding and we have $A_{F}^{ \pm}(f) \sim_{\mathcal{A}} A_{F}^{ \pm}\left(f^{\prime}\right)$.

Since the augmentation of an $\mathcal{A}_{e}$-codimension one map is again codimension one we can augment repeatedly by taking the unfolding $\left(f_{\mu \pm \lambda^{2}}(x), \lambda, \mu\right)$. Thus, define $\left(A_{F}^{+}\right)^{m}(f)$ to be the $m$-fold positive augmentation of $f$ by $F$. For $m>0$ this is the augmentation process repeated $m$ times and for the trivial case $\left(A_{F}^{+}\right)^{0}(f)=f$. Similar definitions can be made for $m$-fold negative augmentation. Note that $A_{F}^{+} A_{F}^{-} f \sim_{\mathcal{A}} A_{F}^{-} A_{F}^{+} f$.

A stable map is called a prism if it is the trivial unfolding of another map. We state the generalisation of Theorem 2.7 of [2] to the case of a real map. (See also Lemma 4.12 and Remark 4.13 of [4].)

Lemma 2.6 (Diminishing Lemma). Suppose that $f:\left(\mathbb{K}^{n}, S\right) \rightarrow\left(\mathbb{K}^{p}, 0\right)$ is an $\mathcal{A}_{e}$-codimension 1 multi-germ such that the miniversal unfolding of $f$ is an m-fold prism. Then there exists a codimension 1 map $h$ with stable unfolding $\left(h_{\lambda}(x), \lambda\right)$ such that $f$ is $\mathcal{A}$-equivalent to $\left(h_{\sum_{i=1}^{m} \pm \lambda_{i}^{2}}(x), \lambda_{1}, \ldots, \lambda_{m}\right)$.

Proof. The proof follows that of Theorem 2.7 of [2] except that the quadratic singularity $\gamma$ is a sum of squares with possibly negative coefficients. The use of Damon's $\mathcal{K}_{V}$-equivalence is justified since his theory works when instead of the discriminant of the real map we take the real part of the discriminant of the complex map as the variety $V$.

It is easy to see that a map in the lemma comes from repeated application of positive and negative augmentation to a primitive $\mathcal{A}_{e}$-codimension 1 map.

3. Classification of mono-germs. In this section we classify corank $1 \mathcal{A}_{e}$-codimension 1 mono-germs.

Theorem 3.1. Suppose that $f:\left(\mathbb{R}^{n}, 0\right) \rightarrow\left(\mathbb{R}^{p}, 0\right), n<p$, is a smooth corank 1 $\mathcal{A}_{e}$-codimension 1 map-germ, then the following are true. 
(i) $f$ is $\mathcal{A}$-equivalent to a map of the form

$$
\begin{aligned}
& \left(u_{1}, \ldots, u_{l-1}, v_{1}, \ldots, v_{l-1}, w_{11}, w_{12}, \ldots, w_{r l}, x_{1}, \ldots, x_{n-l(r+2)+1}, y\right) \\
& \mapsto\left(u_{1}, \ldots, u_{l-1}, v_{1}, \ldots, v_{l-1}, w_{11}, w_{12}, \ldots, w_{r l}, x_{1}, \ldots, x_{n-l(r+2)+1},\right. \\
& \left.\quad y^{l+1}+\sum_{i=1}^{l-1} u_{i} y^{i}, y^{l+2}+\sum_{i=1}^{l-1} v_{i} y^{i}+y^{l} \sum_{i=1}^{n-l(r+2)+1} \pm x_{i}^{2}, \sum_{i=1}^{l} w_{1 i} y^{i}, \ldots, \sum_{i=1}^{l} w_{r i} y^{i}\right),
\end{aligned}
$$

where $r=p-n-1$ and $l+1$ is the multiplicity of the germ. Conversely, any such germ has $\mathcal{A}_{e}$-codimension 1 .

(ii) An $\mathcal{A}_{e}$-versal unfolding is given by unfolding with the addition of the term $\lambda y^{l}$ to the $(p-r l-1)$ th component function, i.e. the term beginning $y^{l+2}$.

(iii) The germ is precisely $(l+2)$-determined.

The proof of Theorem 3.1 is given in the rest of this section.

Remark 3.2. The form is the same as the complex form, see Theorem 3.1 of [5], except that the sum of squares can have negative coefficients.

REMARK 3.3. The squared terms in $x$ and the unfolding parameter term $y^{l}$ show that a map of the above form is an augmentation of the primitive form where $n=l(r+2)-1$.

3.1. Proof of Theorem 3.1 parts (ii) and (iii). As $f$ is finitely $\mathcal{A}$-determined we can assume that $f$ is $\mathcal{A}$-equivalent to some $k$-jet for $k$ large. Parts (ii) and (iii) follow from the fact that if we complexify the $k$-jet, then we get a complex analytic corank 1 $\mathcal{A}_{e}$-codimension 1 map-germ and the results are true in this case, see Theorem 3.1 of [5].

3.2. Proof of Theorem 3.1 part (i). A key element of the classification is the use of the multiple point spaces of the complexified map, so let $f_{\mathbb{C}}$ be the complexification of the map $f$. For the $k$ th multiple point space of a map we would like the set of all $k$-tuples of points of the source that map to the same point in the target. A good method of defining these spaces for corank maps 1, so that they behave well under deformation, is given by Marar and Mond in [7]. We recall this now and generalise it slightly to the case of real maps.

If $G: \mathbb{K}^{n-1} \times \mathbb{K} \rightarrow \mathbb{K}$ is a function in the variables $x_{1}, \ldots, x_{n-1}, y$, then define $V_{i}^{k}(G): \mathbb{K}^{n-1} \times \mathbb{K}^{k} \rightarrow \mathbb{K}$ to be

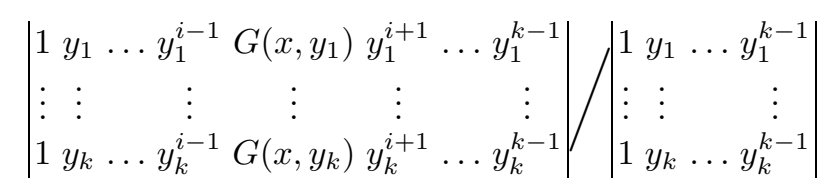

for $i=1, \ldots, k-1$. The group of permutations on $k$ objects, denoted $S_{k}$, acts on $\mathbb{K}^{n-1} \times \mathbb{K}^{k}$ by permutation of the last $k$ coordinates. The function above is $S_{k}$-invariant, and if $G$ is holomorphic then so is $V_{i}^{k}(G)$. When $f$ is a complex analytic map Marar and Mond make the following definition.

Definition 3.4. Suppose $h: \mathbb{C}^{n} \rightarrow \mathbb{C}^{p}, n<p$, is a complex analytic map in the form $h\left(x_{1}, \ldots, x_{n-1}, y\right)=\left(x_{1}, \ldots, x_{n-1}, h_{1}(x, y), \ldots, h_{p-n+1}(x, y)\right)$. Then the $k$ th multiple 
point space, denoted $\widetilde{D}^{k}(h)$, is the set defined in $\mathbb{C}^{n+k-1}$ by $V_{r}^{k}\left(h_{s}\right)=0, r=1, \ldots, k-1$, $s=1, \ldots, p-n+1$.

For a real analytic map $f$ we can make a similar definition for $\widetilde{D}^{k}(f)$ and the defining equations for $\widetilde{D}^{k}\left(f_{\mathbb{C}}\right)$ are just the complexification of those for $\widetilde{D}^{k}(f)$.

The following is shown in the proof in Proposition 3.7 of [6].

TheOrem 3.5. Suppose $f:\left(\mathbb{R}^{n}, 0\right) \rightarrow\left(\mathbb{R}^{p}, 0\right), n<p$, is a corank $1 \mathcal{A}_{e}$-codimension 1 germ, then $\widetilde{D}^{k}\left(f_{\mathbb{C}}\right)$ is non-singular for $k<\operatorname{mult}(f)$ and $\widetilde{D}^{k}\left(f_{\mathbb{C}}\right)$ is quadratic in the variables $\left(y_{1}, \ldots, y_{k}\right)$ for $k=\operatorname{mult}(f)$. The other multiple point spaces are empty.

Now let us return to proving the theorem. First, from Lemma 2.6 we can restrict to the case that $f$ is primitive.

Lemma 3.6. Suppose that $f:\left(\mathbb{R}^{n}, 0\right) \rightarrow\left(\mathbb{R}^{p}, 0\right), n<p$, is a primitive corank 1 $\mathcal{A}_{e}$-codimension 1 map-germ of multiplicity $l+1$. Then,

(i) $l=(n+1) /(p-n+1)$,

(ii) $f$ is $\mathcal{A}$-equivalent to a germ which has $(l+2)$-jet

$$
\begin{aligned}
\left(u, v, w, y^{l+1}+h_{1}(u, v, w) y^{l}+\sum_{i=1}^{l-1} u_{i} y^{i}, y^{l+2}+h_{2}(u, v, w) y^{l}+\sum_{i=1}^{l-1} v_{i} y^{i}\right. & \\
& \left.\sum_{i=1}^{l} w_{1 i} y^{i}, \ldots, \sum_{i=1}^{l} w_{r i} y^{i}\right),
\end{aligned}
$$

where $h_{1}$ and $h_{2}$ are functions in $u, v$ and $w$ with zero constant term.

Proof. (i) The germ $f$ can be unfolded by one parameter to make a corank 1 stable map which is not $\mathcal{A}$-equivalent to the trivial unfolding of another stable map. Hence, the multiplicity is easy to calculate in terms of $n$ and $p$.

(ii) Step 1. Since $f$ has corank 1 it can be put in the form

$$
\left(u, v, w, F_{1}(u, v, w, y), \ldots, F_{r+2}(u, v, w, y)\right),
$$

where $F_{i}(u, v, w, 0)=0$ for $i=1, \ldots, r+2$. On the target we will take coordinates $\left(U, V, W, Y_{1}, Y_{2}, Z_{1}, \ldots, Z_{r}\right)$. To lighten notation these shall remain the labels even after changes of coordinates.

Since $f$ is finitely determined and has multiplicity $l+1$ there exists $i$ such that $F_{i}$ has non-zero constant coefficient for $y^{l+1}$ and without loss of generality we can assume $i=1$. Suppose that the coefficient of $y^{l+1}$ is $h(u, v, w)$ then change coordinates by $Y_{1}^{\prime}=$ $Y_{1} / h(U, V, W)$. Thus we can assume that the coefficient of $y^{l+1}$ in $F_{1}$ is equal to 1 .

From now on we shall assume that we are dealing with the $(l+2)$-jet of $f$, and hence any effects of changes in coordinates will only be noted for this jet. So $f$ is equivalent to a map with $(l+2)$-jet

$$
\begin{aligned}
\left(u, v, w, f_{l+1}(u, v, w) y^{l+2}+y^{l+1}+\sum_{i=1}^{l} f_{i}(u, v, w) y^{i}, \sum_{i=1}^{l+2} g_{i}(u, v, w) y^{i}\right. & \\
& \left.\sum_{i=1}^{l+2} h_{1, i}(u, v, w) y^{i}, \ldots, \sum_{i=1}^{l+2} h_{r, i}(u, v, w) y^{i}\right),
\end{aligned}
$$


for some functions $f_{i}, g_{i}$ and $h_{i, j}$.

We can remove the $y^{l+1}$ terms from the $Y_{2}$ and $Z_{i}$ components by the left change of coordinates:

$$
\begin{aligned}
& Y_{2}^{\prime}=Y_{2}-g_{l+1}(U, V, W) Y_{1} \\
& Z_{i}^{\prime}=Z_{i}-h_{i, l+1}(U, V, W) Y_{1}
\end{aligned}
$$

for all $i=1, \ldots r$.

Step 2. If we complexify the resulting germ, then we get a complex corank 1 codimension 1 germ $f_{\mathbb{C}}$ which by Theorem 3.5 has its multiple point spaces $\widetilde{D}^{k}\left(f_{\mathbb{C}}\right)$ non-singular for $k<l+1$. As noted earlier the defining equations for these multiple point spaces are just the complexification of the real version's equations.

Consider first $\widetilde{D}^{2}\left(f_{\mathbb{C}}\right)$. If this is singular then we can skip this and the next two paragraphs. The defining equations for $\widetilde{D}^{2}\left(f_{\mathbb{C}}\right)$ have linear terms arising from the linear terms in the coefficients of $y$ in $f$. To ensure the non-singularity of $\widetilde{D}^{2}\left(f_{\mathbb{C}}\right)$ the $r+2$ defining equations should have a non-zero linear term. Then there exists a real change of coordinates involving only $u, v$ and $w$ so that (without loss of generality) $f_{1}(u, v, w)=u_{1}$, $g_{1}(u, v, w)=v_{1}$ and $h_{i, 1}=w_{i, 1}$ for $i=1, \ldots r$. Then through a left change of coordinates in $U, V$ and $W$ we can restore the components of $f$ to $(u, v, w, \ldots)$.

When we look at $\widetilde{D}^{3}\left(f_{\mathbb{C}}\right)$ we get $r+2$ equations with non-zero linear terms arising from the coefficients of $y$ in $f$ (i.e. the $u_{1}, v_{1}, w_{i, 1}$ we found above). The other $r+2$ defining equations have linear terms arising from the linear terms of $f_{2}, g_{2}$ and $h_{i, 2}$ and to ensure non-singularity of the multiple point space we have, after a change of coordinates, $f_{2}(u, v, w)=u_{2}, g_{2}(u, v, w)=v_{2}$ and $h_{i, 2}(u, v, w)=w_{i, 2}$ for $i=1, \ldots r$.

Thus by proceeding in this way for all $k<l+1$ we get a map

$$
\begin{gathered}
\left(u, v, w, f_{l+1}(u, v, w) y^{l+2}+y^{l+1}+f_{l}(u, v, w) y^{l}+\sum_{i=1}^{l} u_{i} y^{i}\right. \\
g_{l+2}(u, v, w) y^{l+2}+g_{l}(u, v, w) y^{l}+\sum_{i=1}^{l-1} v_{i} y^{i}, \\
\left.h_{1, l+2}(u, v, w) y^{l+2}+h_{1, l}(u, v, w) y^{l}+\sum_{i=1}^{l-1} w_{1, i} y^{i}, \ldots\right) .
\end{gathered}
$$

Step 3. Now, $\widetilde{D}^{l+1}\left(f_{\mathbb{C}}\right)$ defines a quadratic singularity and so using the facts that its corank is 1 and $V_{l+1}^{l}\left(y^{l+1}\right)$ is linear in $y_{j}$ we can assume that there are $r$ linear coefficients in the $y^{l}$ term of different components. Without loss of generality we can assume that these are the last $r$. Thus we have a map

$$
\begin{gathered}
\left(u, v, w, f_{l+1} y^{l+2}+y^{l+1}+f_{l} y^{l}+\sum_{i=1}^{l} u_{i} y^{i}, g_{l+2} y^{l+2}+g_{l} y^{l}+\sum_{i=1}^{l-1} v_{i} y^{i}\right. \\
\left.h_{1, l+2} y^{l+2}+\sum_{i=1}^{l} w_{1, i} y^{i}, \ldots, h_{r, l+2} y^{l+2}+\sum_{i=1}^{l} w_{r, i} y^{i}\right) .
\end{gathered}
$$

If $g_{l+1}(0,0,0)=0$ then $\widetilde{D}^{l+1}\left(f_{\mathbb{C}}\right)$ would not be quadratic and so we can make the change 
of coordinates $Y_{2}^{\prime}=Y_{2} / g_{l+1}(U, V, W)$. The multiple point spaces of the resulting map will have the same form as $f$, i.e. non-singular, empty or quadratic, and so we can repeat the above working to restore the $v_{i}$.

By making changes of the form

$$
\begin{aligned}
& Y_{1}^{\prime}=Y_{1}-f_{l+2}(U, V, W) Y_{2} \\
& Z_{i}^{\prime}=Z_{i}-h_{i, l+2}(U, V, W) Y_{2},
\end{aligned}
$$

for all $i=1, \ldots, r$, and then restoring the $u, v$ and $w$, we can produce a map of the form in the statement of the lemma.

Lemma 3.7. Suppose $f:\left(\mathbb{R}^{n}, 0\right) \rightarrow\left(\mathbb{R}^{p}, 0\right)$ is $\mathcal{A}$-equivalent to the form

$$
\begin{aligned}
\left(u, v, w, y^{l+1}+h_{1}(u, v, w) y^{l}+\sum_{i=1}^{l-1} u_{i} y^{i}, y^{l+2}+h_{2}(u, v, w) y^{l}+\sum_{i=1}^{l-1} v_{i} y^{i}\right. & \\
& \left.\sum_{i=1}^{l} w_{1 i} y^{i}, \ldots, \sum_{i=1}^{l} w_{r i} y^{i}\right),
\end{aligned}
$$

for some smooth $h_{1}(u, v, w)$ and $h_{2}(u, v, w)$ with zero constant term. Then $f$ is $\mathcal{A}$-equivalent to the primitive $\mathcal{A}_{e}$-codimension 1 map in Theorem 3.1.

Proof. We can make the Tschirnhaus transformation $y^{\prime}=y-\left(h_{1}(u, v, w) /(l+1)\right)$. This removes the $h_{1}(u, v, w) y^{l}$ terms in $Y_{1}$ but takes the other terms out of our preferred form. However, it is easy to calculate that the multiple point spaces of the complexification have the form of Theorem 3.5 and so using the methods in Step 2 of the proof of Lemma 3.6 we can take $f$ into the form

$$
\left(u, v, w, y^{l+1}+\sum_{i=1}^{l-1} u_{i} y^{i}, y^{l+2}+h_{2}(u, v, w) y^{l}+\sum_{i=1}^{l-1} v_{i} y^{i}, \sum_{i=1}^{l} w_{1 i} y^{i}, \ldots, \sum_{i=1}^{l} w_{r i} y^{i}\right),
$$

where $h_{2}$ is possibly different to before, but still with zero constant term.

Let $F$ be the stable map

$$
\left(u, v, w, y^{l+1}+\sum_{i=1}^{l-1} u_{i} y^{i}, y^{l+2}+\lambda y^{l}+\sum_{i=1}^{l-1} v_{i} y^{i}, \sum_{i=1}^{l} w_{1 i} y^{i}, \ldots, \sum_{i=1}^{l} w_{r i} y^{i}, \lambda\right) .
$$

Let $L(U, V, W)$ be the linear part of $h_{2}(U, V, W)$ and define the family of maps $g_{t}(U, V, W, Y, Z)=\left(U, V, W, Y, Z, L(U, V, W)+t\left(h_{2}(U, V, W)-L(U, V, W)\right)\right)$. Thus, the pull-back $g_{1}^{*}(F)$ induces $f$.

We now employ Damon's theory of $\mathcal{K}_{V}$-equivalence, see [3]. Let $V=F\left(\mathbb{C}^{n+1}\right)$ and $\operatorname{Derlog}(V)$ be the liftable vector fields over $F$. If $g:\left(\mathbb{C}^{p}, 0\right) \rightarrow\left(\mathbb{C}^{p+1}, 0\right)$ induces $h$ as a pull-back, then

$$
N \mathcal{A}_{e} h \simeq N \mathcal{K}_{V, e} g:=\frac{\theta(g)}{\operatorname{tg}\left(\theta_{\mathbb{C}^{p}}\right)+g^{*}(\operatorname{Derlog}(V))} .
$$

By Nakayama's Lemma we have

$$
t g_{t}\left(m_{\mathbb{C}^{p}} \theta_{\mathbb{C}^{p}}\right)+g_{t}^{*}(\operatorname{Derlog}(V))=m_{\mathbb{C}^{p+1}} \theta\left(g_{t}\right)
$$


if and only if

$$
t g_{t}\left(m_{\mathbb{C}^{p}} \theta_{\mathbb{C}^{p}}\right)+g_{t}^{*}(\operatorname{Derlog}(V))+m_{\mathbb{C}^{p}}^{2} \theta\left(g_{t}\right)=m_{\mathbb{C}^{p+1}} \theta\left(g_{t}\right) .
$$

Thus, the codimension of $g_{t}^{*}(F)$ depends only on the linear part of $h_{2}$. If $h_{2}$ is linear, then it is possible to show by calculation that the $\mathcal{A}_{e}$-tangent space has the same form as Theorem 3.5 of [5] (see also [2]) and hence has codimension 1. Therefore, all $g_{t}^{*}(F)$ are codimension 1 . The family members are all $\mathcal{K}$-equivalent and so by Lemma 2.3 they are all $\mathcal{A}$-equivalent. So $f$ is equivalent to $g_{0}^{*}(F)$ and, in effect, we can assume that $h_{2}(U, V, W)$ is linear.

Now we take the family of maps indexed by $t$ and produced from the above $f$ by replacing $h_{2}(u, v, w)$ with $t L(u, v, w)$ (and taking $h_{1}(u, v, w)=0$ of course). This family can be induced from the stable map $F$ by the family $(U, V, W, Y, Z) \mapsto$ $(U, V, W, Y, Z, t L(U, V, W))$. Since the $\mathcal{A}_{e}$-codimension for each member is 1 and all the members are obviously $\mathcal{K}$-equivalent, by Lemma 2.3 they are all $\mathcal{A}$-equivalent. In particular $f$ is $\mathcal{A}$-equivalent to the standard form given by $t=0$.

We now finish the proof of the main theorem.

Proof of Theorem 3.1(i). By Lemma $2.6 f$ is $\mathcal{A}$-equivalent to the repeated augmentation of an $\mathcal{A}_{e}$-codimension 1 map $f^{\prime}$, where $f^{\prime}$ is primitive. The map $f^{\prime}$ is $\mathcal{A}$-equivalent to a map with $(l+2)$-jet of the form in Lemma 3.6. By Lemma 3.7 the $(l+2)$-jet is equivalent to the primitive normal form in the statement of the theorem, which is $(l+2)$-determined by part (iii). Thus $f^{\prime}$ is $\mathcal{A}$-equivalent to the primitive normal form.

\section{References}

[1] T. Cooper, Map germs of $\mathcal{A}_{e}$-codimension one, Ph.D. Thesis, University of Warwick, 1993.

[2] T. Cooper, D. Mond, R. Wik Atique, Vanishing topology of codimension 1 multi-germs over $\mathbb{R}$ and $\mathbb{C}$, Compositio Math. 131 (2002), 121-160.

[3] J. Damon, $\mathcal{A}$-equivalence and the equivalence of sections of images and discriminants, in: Singularity Theory and its Applications, Part I, Lecture Notes in Math. 1462, Springer, Berlin, 1991, 93-121.

[4] J. Damon, On the legacy of free divisors: discriminants and Morse-type singularities, Amer. J. Math. 120 (1998), 453-492.

[5] K. Houston, On the classification of complex multi-germs of corank one and codimension one, University of Leeds Preprint 2003, http://www.amsta.leeds.ac.uk/ khouston/psfiles/cod1.ps

[6] K. Houston, A note on good real perturbations of singularities, Math. Proc. Cambridge Philos. Soc. 132 (2002), 301-310.

[7] W. L. Marar, D. Mond, Multiple point schemes for corank-1 maps, J. London Math. Soc. (2) 39 (1989), 553-567.

[8] J. N. Mather, Stability of $C^{\infty}$ mappings IV. Classification of stable germs by $\mathbb{R}$-algebras, Inst. Hautes Études Sci. Publ. Math. 37 (1969), 223-248.

[9] D. Mond, On the classification of germs of maps from $\mathbb{R}^{2}$ to $\mathbb{R}^{3}$, Proc. London Math. Soc. (3) 50 (1985), 333-369.

[10] C. T. C. Wall, Finite determinacy of smooth map-germs, Bull. London Math. Soc. 13 (1981), 481-539. 